\title{
An inducible transposon system to terminate the function of a selectable marker in transgenic plants
}

\author{
Yuh-Chyang Charng $\cdot$ Kuan-Te Li $\cdot$ \\ Hung-Kuang Tai $\cdot$ Na-Sheng Lin $\cdot$ Jenn Tu
}

Received: 5 June 2007/Accepted: 23 September 2007/Published online: 15 November 2007

(C) Springer Science+Business Media B.V. 2007

\begin{abstract}
Since the maize transposon $A c$ can move to a new location within the genome, it has been used in removing selectable markers in transgenic plants. In this paper, we developed an inducible transposon system to truncate a selectable marker in transgenic plants. In this system, the marker gene was accompanied by the inducible transposon, but one end of the transposon was located in the intron of the marker gene. As an example of a marker gene, we isolated the rice 5-enolpyruvylshikimate-3-phosphate synthase (epsps) and modified it for glyphosate tolerance. The transposon contained $A c$ transposase, which fused with the promoter of the inducible gene for pathogenesis-related protein 1a (PR1a). This construct was engineered into an expression vector pCAMBIA1300, harboring a hygromycin-resistant gene. The construct was first transformed into rice calli, and transformed plants were selected on hygromycin-containing medium. The stably transformed calli underwent determination for normal transcripts and
\end{abstract}

Electronic supplementary material The online version of this article (doi: 10.1007/s11032-007-9137-3) contains supplementary material, which is available to authorized users.

Y.-C. Charng $(\bowtie) \cdot$ K.-T. Li · H.-K. Tai

Department of Agronomy, National Taiwan University,

No. 1 Sec. 4 Roosevelt Rd., Taipei, Taiwan,

Republic of China

e-mail: bocharng@ntu.edu.tw

N.-S. Lin $\cdot$ J. Tu

Institute of Plant and Microbial Biology, Academia

Sinica, Taipei, Taiwan, Republic of China tolerance to glyphosate. The results were applied to a rice transformation with the same construct, but using glyphosate as the selective agent. By determining the transformation efficiency, T-DNA copy patterns, we demonstrate that the modified epsps could be a suitable selectable marker to create transgenic rice. Furthermore, after obtaining stable transgenic plants and inducing transposition by salicylic acid, the transposon was excised, the marker gene became truncated, and its expression was terminated. This strategy could be applicable to yield self-stabilizing transposon by locating the transposon's end in the transposase gene's intron.

Keywords Ac transposase - epsps .

Inducible transposon - Selectable marker .

Transgenic plants

$\begin{array}{ll}\begin{array}{l}\text { Abbreviations } \\ \text { epsps }\end{array} & \begin{array}{l}\text { 5-enolpyruvylshikimate-3-phosphate } \\ \text { synthase gene }\end{array} \\ \text { GOI } & \text { Gene of interest } \\ \text { SA } & \text { Salicylic acid } \\ \text { TPase } & \text { Transposase gene }\end{array}$

\section{Introduction}

The genetic modification of plants offers improvements in agricultural practices, food safety, and human health. The development of transgenic plants 
requires the use of selectable marker genes, because the efficiency of plant transformation is less than optimal for many important plant species (Hohn et al. 2001). In current plant transformation systems, a selectable marker gene is co-delivered with the gene of interest (GOI) to identify and separate rare transgenic cells from non-transgenic cells.

Usually, a conditional dominant gene, with no influence on the growth or morphology of plants, is used as a selectable marker (Endo et al. 2002). Dominant genes encoding either antibiotic or herbicide resistance are widely used as selectable markers. The antibiotics and herbicides used to select rare transgenic cells from non-transgenic cells generally have negative effects on proliferation and differentiation (Ebinuma et al. 1997). For example, glyphosate inhibits 5-enolpyruvylshikimate-3-phosphate synthase (EPSPS; E. C. 2.5.1.19), a critical enzyme in the shikimate pathway for the biosynthesis of aromatic amino acids (Bradshaw et al. 1997; Schönbrunn et al. 2001). Glyphosate-resistant epsps was successfully used as a selectable marker in the plant transformation of oilseed rape, soybean, potato, maize, and wheat (Clemente et al. 2000; Hu et al. 2003; Miki and McHugh 2004; Zhou et al. 1995).

Over the past few years, consumer and environmental groups have expressed concern about the use of markers from an ecological and food safety perspective. The development of marker-free transgenic plants is desirable in agricultural biotechnology. Many strategies to produce markerfree transgenic plants have been described (reviewed by Ebinuma et al. 2001; Hare and Chua 2002; Hohn et al. 2001; Miki and McHugh 2004). The Cre-lox site-specific recombination system has been widely studied for marker removal. In fact, the first markerfree commercial transgenic plant was developed using Cre-lox technology (Ow 2007). In the transformation vector, the marker is flanked by directly oriented lox sites. A cre gene is introduced into the genome from a genetic cross. The expression of the Cre protein causes recombination between the two lox $\mathrm{P}$ sites, and the marker is lost during the process of recombination. New techniques have been developed to control the cre gene expression by flanked with an inducible promoter (Zuo et al. 2001). A limitation of this system is that the high level of expression of the cre gene may result in phenotypic aberrations in some plant species (Hajdukiewicz et al. 2001).
In contrast to the Cre-lox recombination system leading to the loss of the marker gene, the transposon system (e.g., $A c / D s$ ) offers information about the new location of the removed marker's DNA. The maize transposon $A c$ is an autonomous transposable element of $4565 \mathrm{bp}$ and is active in a wide range of plant species. It codes for a single gene product, Ac transposase, which, together with the inverted repeats and about $250 \mathrm{bp}$ of both ends (terminal regions) of the transposon and putative host factors, is the only prerequisite for transposition of the Ac element in plants (Haring et al. 1991). In the transformation vector, the marker gene is inserted into the $D s$ element. The expression of the $A c$ transposase excises both ends of the transposon and usually re-integrates into other locations on the chromosome. When the transposon transposes within the same chromosome (linked transposition), both insertion sites of the TDNA (harboring the marker gene) and the transposon (harboring the GOI) need regulatory approval for commercialization. With unlinked transposition, the marker gene can be removed by out-crossing. Although the work is time consuming, all removed information remains clear for regulatory approval. Furthermore, with the transposon system, one successful transformation can create more independent transgenic lines because of the re-integrated loci. This feature is valuable for creating transgenic plants in species with low transformation efficiency. However, out-crossing with this system cannot be used with vegetatively propagated plants and woody tree species. According to this, we designed a system to truncate a marker gene after transposition, termed "marker-off", which respects the marker-free system and does not require segregating the marker away. The system involves introducing an intron-containing marker gene, accompanied by a transposon whose one end is located in the intron of the marker gene, into a transformation vector. The prerequisite of the strategy is to obtain an intron-containing marker gene. Since Howe et al. (2002) successfully modified the maize epsps as a selectable marker for creating transgenic maize, we expected that a modified rice (Oryza sativa L.) epsps could serve as a selectable marker for the transformation of rice. For the transposon to terminate the marker, we applied a chemical inducible transposon. Previously, the $A c$ transposase gene was fused with the promoter of the pathogenesis-related protein 1a (PR-1a). PR-1 
proteins are induced in plants as a consequence of the hypersensitive defense reaction elicited by pathogen infection (van Loon and van Kammen 1970), by exogenous application of some chemicals, e.g., salicylic acid (SA) (White 1979), and by developmental stimuli (Gruener and Pfitzner 1994). This PR1a-transposase fusion was inserted in a $D s$ element to yield an inducible transposon (Charng et al. 2000). Here, the $5^{\prime}$ end of this inducible transposon was constructed in the first intron of epsps. We studied the expression pattern of the modified epsps and glyphosate resistance activity in transgenic rice, and then determined the rice transformation efficiency and TDNA integration copies when glyphosate was used as the selection agent. Finally, we induced transposition events to terminate the glyphosate-tolerance ability in transgenic rice.

\section{Materials and methods}

\section{Modification of the rice epsps}

Plasmid p6140 containing a genomic copy of epsps from rice was a gift from Dr. Kishima (Hokkaido University, Japan). To construct a gene for the glyphosate-tolerant form of the EPSPS enzyme, we used site-directed mutagenesis to change two codons in the coding sequence as described previously (Howe et al. 2002), whereby the authors modified the corn epsps cDNA clone for a glyphosate-tolerant form. The DNA fragment containing exons 2-4 was firstly sub-cloned into plasmid pBC. The codon for the glycine residue at position 168 was changed to encode an alanine residue and that for the glycine residue at position 211 was changed to encode an aspartic acid residue by polymerase chain reaction (PCR) as described. The resulting DNA fragment, together with the other epsps exons, was used for subsequent construction.

DNA manipulation and plant transformation

The construction procedures for the KCEH system ( $K C E H$ transposon plus the marker gene) is depicted in the supplementary material. All cloning and DNA manipulations followed standard procedures (Sambrook and Russel 2001) with the use of chemicals from Roche (Basel, Switzerland). All transformations involved the use of rice (Oryza sativa L. cv. TNG67) as described (Toki 1997).

Assay of T1 progeny resistant to glyphosate

Successful transgenic plants resistant to glyphosate were self-pollinated to obtain $\mathrm{T} 1$ seeds. The seeds were imbibed in flowing water for 2 days, then transferred to an iron grid and kept in water for 2 weeks, and then soil for another week. The three leaf-age stage rice seedlings were sprayed once with 5,000 ppm Roundup ${ }^{\circledR}$. The effect could be observed after 1 week.

RT-PCR analysis of epsps expression

RNA extracted from the transgenic rice calli was reverse transcribed with use of the SuperScript ${ }^{\mathrm{TM}}$ First-Strand Synthesis System (Invitrogen). Primers specific to each of the two target messages were used in subsequent PCR amplification: R-E1FC (5'-ATCGTGCTCCAG CCCATCAG- $\left.3^{\prime}\right)$, mKRT1F (5'-CAACTCTTCTTGG GGAACGCTGCT-3'), and mKRT2R (5'-CAAGGAA ACAGTCGACATCCGCGT-3'). Primers specific to endogenous epsps were KRT1F (5'-CAACTCTTCT TGGGGAACGCTGGA-3') and KRT2R (5'-CA AGGAAACAGTCGACATCCGCAC- $3^{\prime}$ ).

Induction of $K C E H$ transposition and the determination of insertion sites

For induction experiments, T1 rice seeds of each transformed line harboring a single copy of $K C E H$ were incubated on callus induction medium (CIM) containing hygromycin for 4 weeks in order to yield enough calli for the induction experiments. We previously found $5 \mathrm{mM}$ of SA to have the highest induction efficiency in transgenic rice containing a PR-1a-based transposon (Charng et al. 2007), so we induced transposition by incubating transgenic rice calli harboring the KCEH system on CIM containing $5 \mathrm{mM}$ of SA. The empty donor sites of transposition were determined with the primers CAMBIA1 $\left(5^{\prime}\right.$ GTCGACTTTCTAGAGGATCCG-3') and mKRT2R (5'-CAAGGAAACAGTCGACATCCGCGT-3'). 
The flanking sequences of the $K C E H$ element in transgenic plants were amplified by TAIL-PCR (Liu et al. 1995; Sha et al. 2004) with the following oligonucleotide primers: $3-1$ (5'-GTGTGCTCCAGATT TATATGG-3'), 3-2 (5'-GATTTCGACTTTAACCCG ACCGGA-3'), and 3-3 (5'-CGTTTTCGTTACCGG TATATCCCG- $3^{\prime}$ ) for the $3^{\prime}$ end. The arbitrary degenerate (AD) primers and TAIL-PCR were as described previously (Liu et al. 1995; Sha et al. 2004), with following modification: the primary TAIL-PCR contained approximately $150 \mathrm{ng}$ of rice genomic DNA.

\section{Results}

Construction of the transposon-mediated markeroff system

The construction of the inducible transposon system to truncate the marker gene is depicted in Fig. 1a. First, the $5^{\prime}$ end of the $A c$ element was inserted in the first intron of the rice genomic epsps driven by the CaMV35S promoter, which had been modified to produce a glyphosate-tolerant form of the enzyme. To complete a new transposon, a PR-1a::TPase fusion was flanked by the $3^{\prime}$ end of $A c$. The new transposon, $K C E H$, contains a PR-1a::TPase fusion, a CaMV35S promoter, and the first exon of the modified epsps. The KCEH system (KCEH transposon plus the marker gene) was inserted into the binary vector pCAMBIA 1300, yielding the plasmid pKCEH, which was introduced into the Agrobacterium tumefaciens strain LBA4404 for plant transformation.

Expression of the modified epsps in transgenic rice plants

To determine the behavior of the modified epsps in rice, the KCEH construct was transformed into rice, with hygromycin used as the selection agent. A total of 21 single-copy transgenic lines were determined by PCR and DNA blot analysis (data not shown), and the total RNA of each line was extracted for RT-PCR. The modified epsps in the KCEH construct and endogenous rice epsps differ in $4 \mathrm{bp}$. To eliminate the possibility of amplifying the endogenous rice epsps, we designed two primers, mKRT1F and mKRT2R,
Fig. 1 (a) Schematic representation of the $\mathrm{KCEH}$ system and the location of primers (shown as solid triangles). $\mathrm{LB}=$ left border; $\mathrm{RB}=$ right border; $5^{\prime}$ and $3^{\prime}=A c$ left and right terminalinverted repeat; PR-

$1 \mathrm{a}=$ inducible promoter;

TPase $=$ transposase gene;

$35 \mathrm{~S}=$ cauliflower mosaic virus 35S RNA promoter;

$1 \sim 8=$ epsps exon $1 \sim$ exon 8 ; bold lines = epsps introns; *mutation site. (b) Reverse transcriptase polymerase chain reaction (RT-PCR) analysis of modified (or endogenous) epsps expression in transgenic rice (lanes 4, 5, 8, 9) or wild type (lanes 2, 3, 6, 7) with primers R-E1FC and mKRT2R (lanes 2 and 4), mKRT1F and mKRT2R (lanes 3 and 5), R-E1FC and KRT2R (lanes 6 and 8), KRT1F and KRT2R (lanes 7 and 9). Lane 1: 100-bp marker a
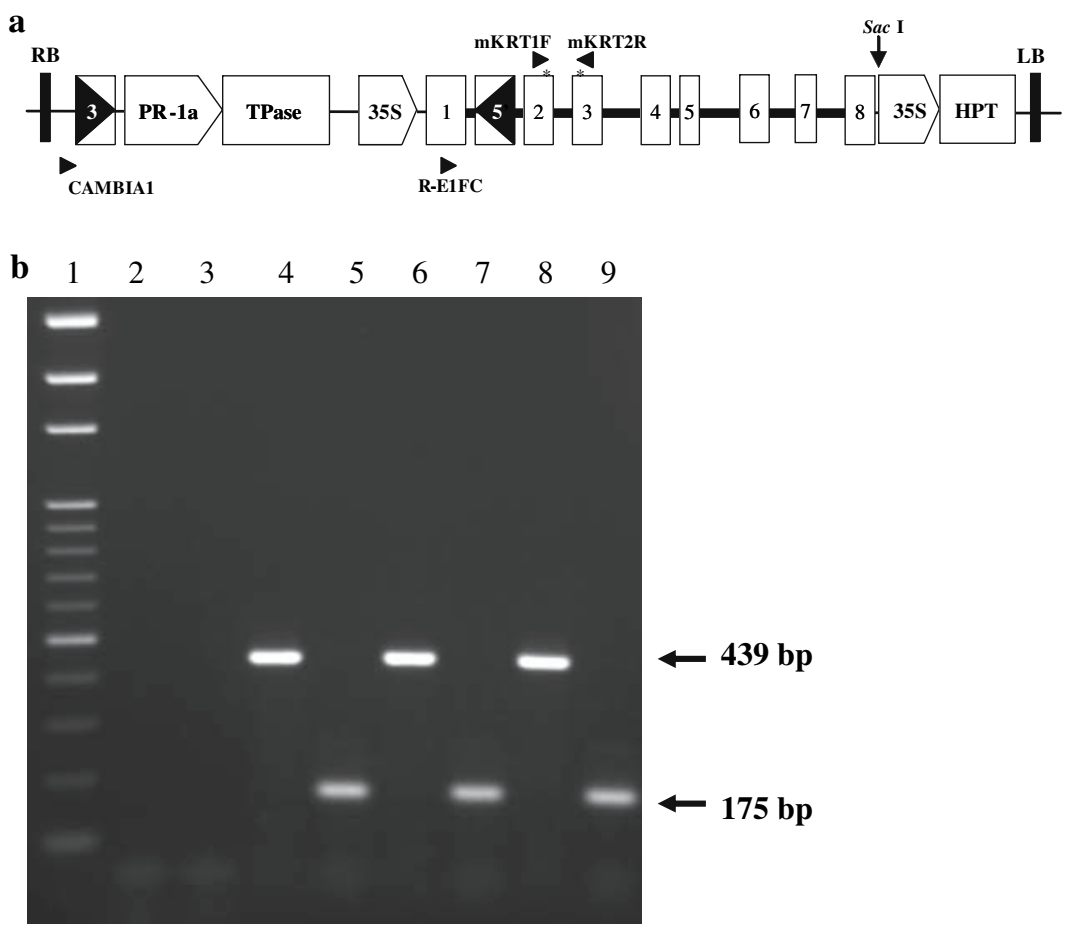
Fig. 2 Determination of the glyphosate concentration of the modified epsps as a selective marker. Each line was cultured in callusinduced medium under different concentrations of glyphosate $(1,5$, and $10 \mathrm{mM}$ ) for 5 weeks. KCEH-H1 and KCEH-H6 are independent transgenic lines with hygromycin used as the selection agent. TNG67, wild-type rice

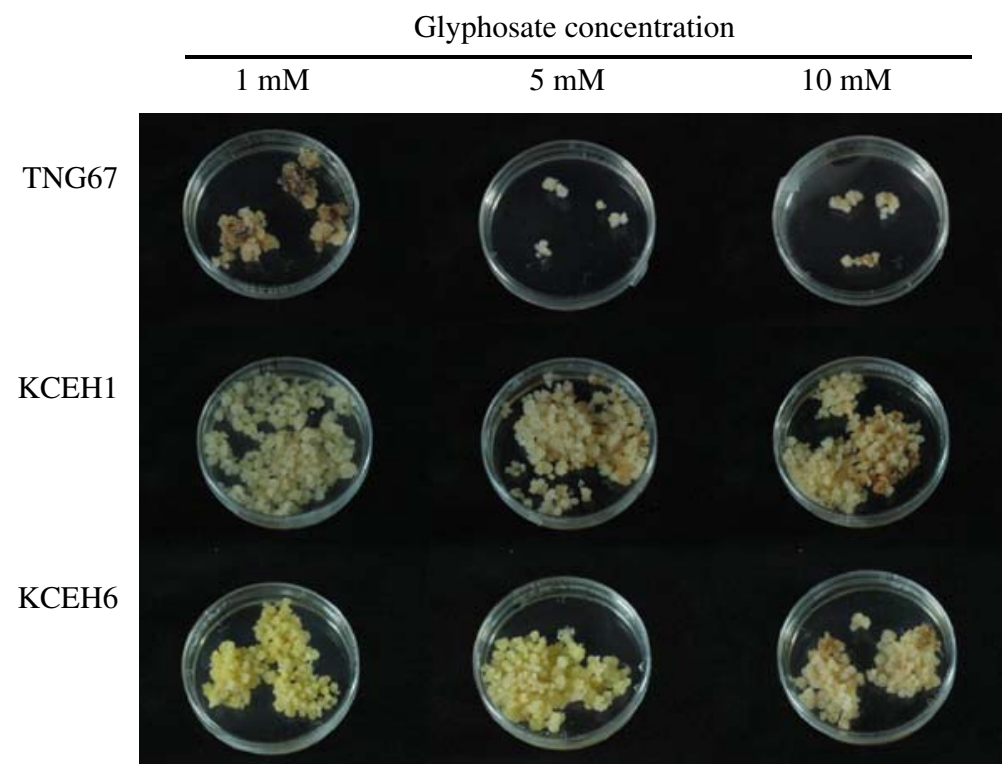

which were specific to the modified sites of epsps. These two primers are mismatched at the two $3^{\prime}$ terminal bases for endogenous epsps and have more power to identify transgenes. To ensure functional splicing of the first intron by PCR, the primers RE1FC and mKRT2R were designed from the first and third exons of the modified epsps. In normal splicing of the endogenous epsps, the predictive fragment is 439 bp (Fig. 1b). With mKRT1F and mKRT2R, only modified epsps could be amplified by PCR, and the predicted fragment 175 bp was amplified (Fig. 1b). The experiments were performed again but primers mKRT1F and mKRT2R were replaced with primers KRT1F and KRT2R, which are matched for endogenous epsps. With these two primers, the expected DNA fragments could be amplified in transgenic rice, as well as in wild type TNG67.

Efficient Agrobacterium-mediated transformation of rice with modified epsps

To determine whether the modified epsps can be used as a selectable marker in rice, the regenerated calli of transgenic lines containing a single copy of the modified epsps and the wild type were incubated with CIM containing glyphosate $(1,5$, or $10 \mathrm{mM}$ ) for 4 weeks. Figure 2 shows the tolerance of transgenic calli lines up to $10 \mathrm{mM}$ of glyphosate, with the growth of wild-type calli extremely restricted on media containing $5 \mathrm{mM}$ or $10 \mathrm{mM}$ of glyphosate. Thus, we used $5 \mathrm{mM}$ of glyphosate as the selection condition for rice transformation.

The pKCEH construct was introduced into the embryonic calli of rice, and transgenic plants were screened with use of $5 \mathrm{mM}$ of glyphosate. The transgenic calli differed from nontransgenic calli by their proliferation potential under glyphosate treatment (Fig. 3a). The transgenic calli were transferred to differentiation medium to set shoots (Fig. 3b), then, the resulting plantlets were transferred to the soil and grown in the greenhouse to set seeds (Fig. 3c). We obtained 98 transgenic lines derived from 1,086 calli pools, for $9.02 \%$ transformation efficiency. To verify stable transformation, Sac Idigested genomic DNA from each independent transgenic line underwent Southern blot analysis. Since the modified epsps differs from the endogenous epsps by only $4 \mathrm{bp}$, we used the 1.1-kb fragment of the hpt gene as a probe. As an example, in Fig. 4, 9 of 20 transgenic lines contained only one T-DNA copy and the others more than two copies. From 98 transgenic lines, 43 single-copy independent transgenic rice lines were obtained, which indicates that the selection procedure is suitable for creating singlecopy lines. To determine the inheritance of the modified epsps and whether the transgenic rice plants were resistant to Roundup ${ }^{\circledR}$ (glyphosate applied in the field), mature rice seedlings from the T1 progeny were sprayed with $5,000 \mathrm{ppm}$ Roundup ${ }^{\mathbb{R}}$. We 
Fig. 3 Different periods in the modified rice epsps transformation system. (a) Proliferation of calli in 5-mM of glyphosate growth medium over 5 weeks (yellow arrows).

(b) Differentiation of proliferating calli into shoots and roots in shooting medium over 5 weeks. (c) Transformants were transferred to soil for 2 months, grew well, and headed normally (red box)
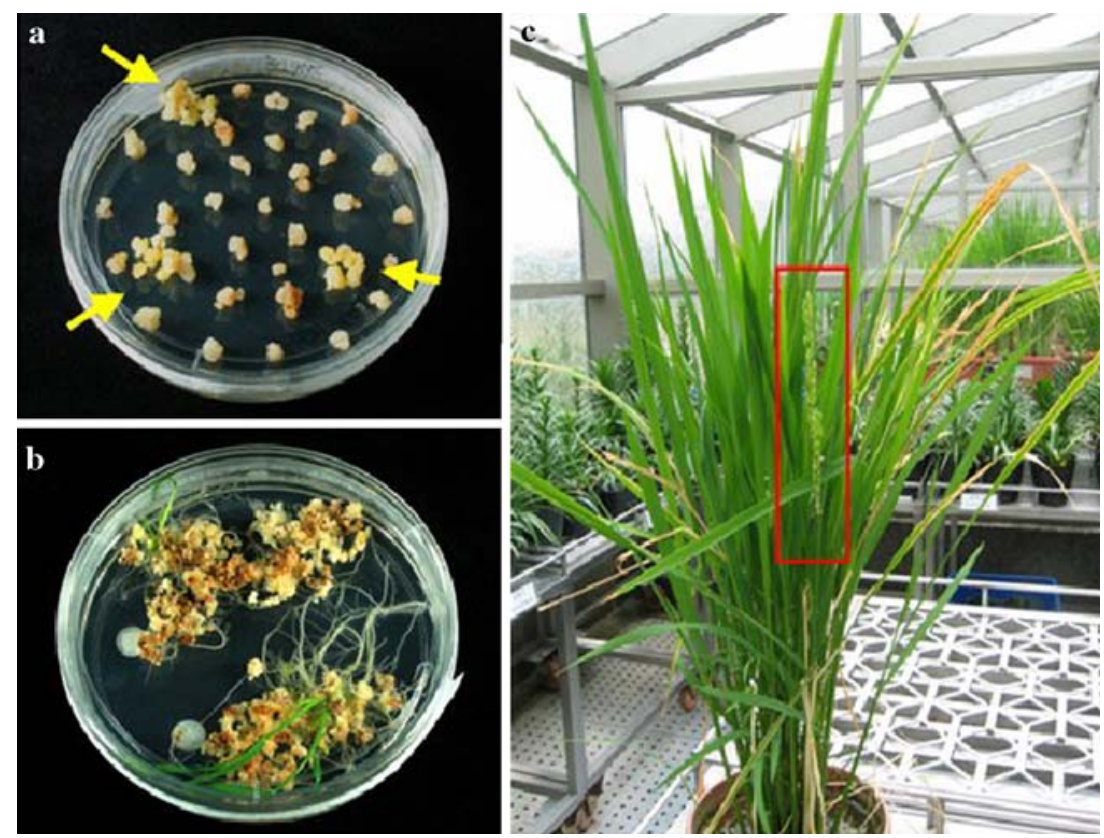

observed the expected 3:1 Mendelian ratio for the modified epsps among all single-copy T-DNA transgenic lines (data not shown; another progeny assay result is described below). Thus, the glyphosatetolerant phenotype was inherited as a single Mendelian locus in all of the plants tested. All of these results indicate that the modified epsps is suitable as a selectable marker in rice.

Transposition of $K C E H$ and termination of the glyphosate-tolerant epsps in transgenic rice

Previously, we introduced an $A c$-based inducible transposon, INAc, into rice and found that the highest transposition efficiency was induced with $5 \mathrm{mM}$ of SA (Charng et al. 2007). Thus, to remove the functional glyphosate-tolerant epsps, we applied $5 \mathrm{mM}$ of SA to transgenic rice calli to trigger the $K C E H$ transposon. Calli regenerated from the T1 rice seeds of each transformed line harboring a single copy of $K C E H$ were incubated on CIM containing $5 \mathrm{mM}$ of SA to induce transposition. The excision events were determined by PCR with the primers mKRT2R and CAMBIA1. To determine the empty donor site, a 550-bp DNA fragment was expected (Fig. 5a). Of 43 single-copy lines, seven transgenic lines yielded the expected 550-bp DNA products, for
$16 \%$ somatic transposition efficiency. Sequencing analysis confirmed the residual DNA after the excision of the transposon (Fig. 5b).

According to our previous studies of transposition events in rice, $A c$-based inducible transposons are very active in induced rice calli, but, sometimes, only a portion of cells contain the empty donor site (partial transposition events; Charng et al. 2007). We determined whether the transposition events passed through the germ line and were inherited in the progeny (germinal transposition) or not (somatic transposition). The remaining calli of the transposed lines, as well as non-SA-treated calli (controls), were cultured to set shoots and then transplanted to soil for self-pollination. The seedlings of the progeny underwent PCR to determine the inheritance of the transposition events. Of seven lines showing transposition, only two showed the transposition events inherited in the progeny. The seedlings of the progeny were treated with Roundup ${ }^{\circledR}$ as described above. All 25 seedlings were glyphosate-sensitive, which indicates the loss of the glyphosate-tolerant function (Fig. 6 left). As a control, siblings of the same transgenic line which had not been induced with SA for transposition were cultured to harvest self-pollinated seeds for glyphosate-tolerant assay and showed glyphosate-tolerance as a single Mendelian locus pattern (Fig. 6 right). 


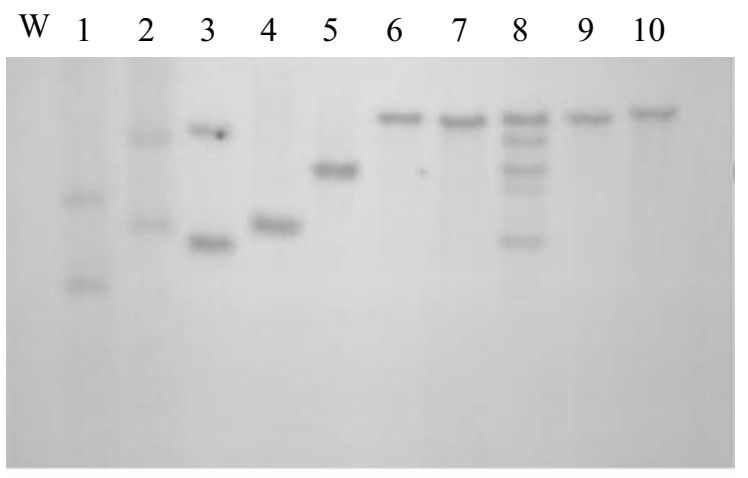

$\begin{array}{lllllllllll}\mathrm{W} & 11 & 12 & 13 & 14 & 15 & 16 & 17 & 18 & 19 & 20\end{array}$

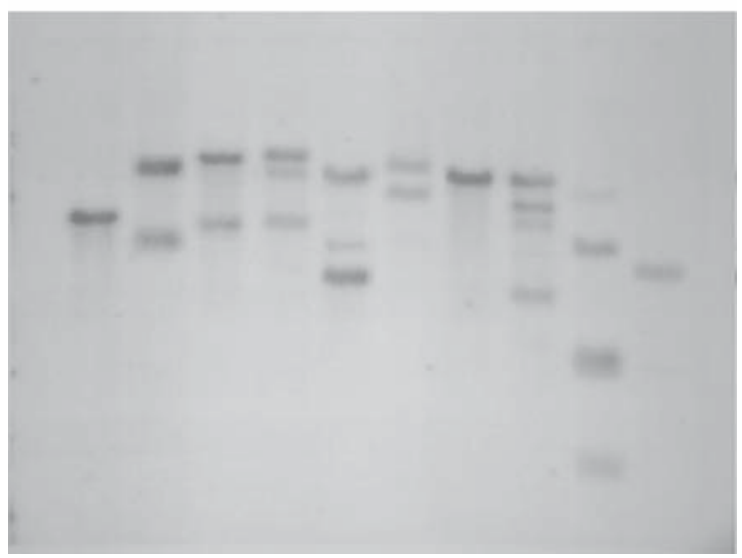

Fig. 4 Analysis of T-DNA copies of transgenic rice selected by $5 \mathrm{mM}$ of glyphosate. Instead of the use of modified epsps, $h p t$ was used as a probe for DNA blot analysis because of the presence of endogenous epsps. W=wild type TNG67 rice; 1-20 represent the selected plants from $\mathrm{T} 0$ glyphosate-tolerant transformants. The single-copy T-DNA transgenic lines were re-numbered for subsequent analysis

Genomic DNA containing the independent transposed $K C E H$ was collected to amplify the flanking sequences of the transposed $K C E H$ elements. The flanking sequences were isolated by TAIL PCR. A summary of the significant homologies obtained after comparison of the flanking sequences from a public database is shown in Table 1. Of six independent transposition events, four showed linkages and one no linkage to the T-DNA locations. For one event, the flanking sequence was not obtained, possibly because of failure to amplify the PCR product or loss of the transposon after excision from its donor site. Taken together, the results indicate that the $\mathrm{KCEH}$ system offers a desirable selectable marker for rice transformation and the ability to remove the marker thereafter.

\section{Discussion}

Marker-free systems investigated in transgenic plants include the use of co-transformed genes, site-specific recombination, and transposon mediation. Co-transformed and transposon-mediated systems enable the GOI and marker to integrate into different loci in the plant genome. Unlinked marker genes can then be segregated away from the GOI to produce markerfree transgenic plants (reviewed by Ebinuma et al. 2001), but this technology is not useful for woody plants or plants that reproduce vegetatively. Sitespecific recombination systems remove the marker gene by the use of single enzymes (e.g., Cre) acting on specific target sequences. Each of the target sites contain a few oligonucleotides surrounded by a few inverted repeats to determine the orientation of the target site. The expression of the recombinase causes recombination between the target sites and results in the loss of the marker gene flanked by the sites. However, high levels of recombinase expression may result in genome rearrangements at cryptic-target sites. Although such sites have not been described in the nuclear genomes of plants, chloroplast cryptic lox sites have been described (Hajdukiewicz et al. 2001). In contrast to the short target recognition sequences (e.g., 34 bp for loxP sites), the existence of pseudotarget sites for transposons (e.g., about $250 \mathrm{bp}$ for Acl $D s)$ is believed to be rare in the plant genome. We, therefore, applied an inducible transposon technology to develop a marker-off system without the need for out-crossing. The new system is called KCEH and contains the $A c$-based inducible transposon $\mathrm{KCEH}$ and the marker gene, modified epsps. We inserted one end of $\mathrm{KCEH}$ into the first intron of the marker and then introduced it into the rice genome; epsps was expressed functionally (Figs. 1, 2, and 6). Yet, when the transposon was excised after induced transposition, the DNA sequences within both ends were removed and the marker was truncated and lost its function.

To study the KCEH system, we first determined whether the modified epsps was a useful marker. Although the first intron was inserted by the $5^{\prime}$ end of the transposon, Fig. $1 \mathrm{~b}$ and the sequence analysis (data not shown) indicated that the splice junction of the first intron could be identical to the junction of the native epsps. Figure 2 demonstrated the glyphosatetolerance function of the modified epsps in transgenic 
Fig. 5 Analysis of transposition events of the SA-induced transgenic rice harboring $\mathrm{KCEH}$ system.

(a) PCR analysis of $\mathrm{KCEH}$ transposition with the primers CAMBIA1 and mKRT2R and the expected fragments. (b) Sequence of the empty donor site of the transposition events,

leaving the sequences from CAMBIA vector (italics) and the truncated epsps, in which the exons are shown in bold. The primers

CAMBIA1 and mKRT2R are indicated as arrows. $\mathrm{M}=100$-bp marker a

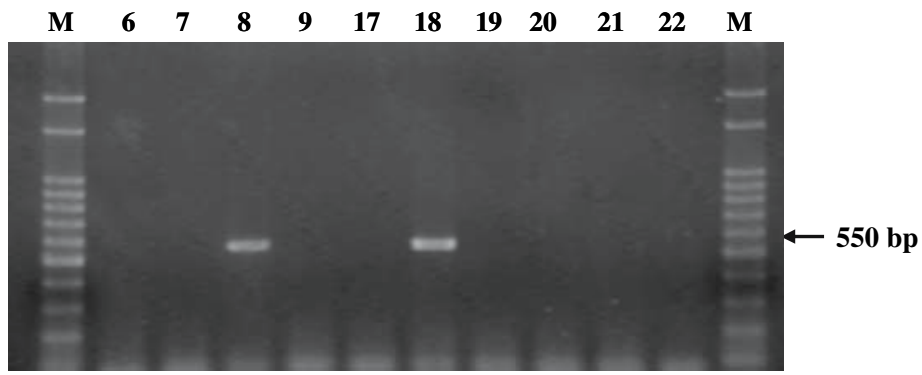

b

CAMBIA1

$\overrightarrow{\text { GTCGACTTTCTAGAGGATCCGC } G C G G C C G G G T C A C G G C G A T C T A A T C G}$ AATTCCCGCGGCCGCTATTATTTCAAGCGGGTATTGATCCTTTGACA TGTGATTGATCATTTTTTTTTCTCTGGTTATTAGGGCACAACAGTGG TGGACAACTTGCTGAACAGTGAGGATGTTCACTACATGCTTGAG GCCCTGAAAGCCCTCGGGCTCTCTGTGGAAGCAGATAAAGTTG CAAAAAGAGCTGTAGTCGTTGGCTGTGGTGGCAAGTTTCCTGTT GAGAAGGATGCGAAAGAGGAAGTGCAACTCTTCTTGGGGAACG CTGCTACTGCAATGCGACCATTGACAGCAGCCGTGACTGCTGCT GGTGGAAATGCAACGTATGTTTTTTTTTTTAATGTTTATGAAAATAT GTATGGAATTCATGGGGTATGTTTTATGACCTTTTTCTTTACCATCA GTTATGTGCTTGATGGAGTGCCACGAATGAGGGAGAGACCGAT TGGTGACTTGGTTGTCGGGTTGAAACAACTTGACGCGGATGTC GACTGTTTCCTTG KRT2R
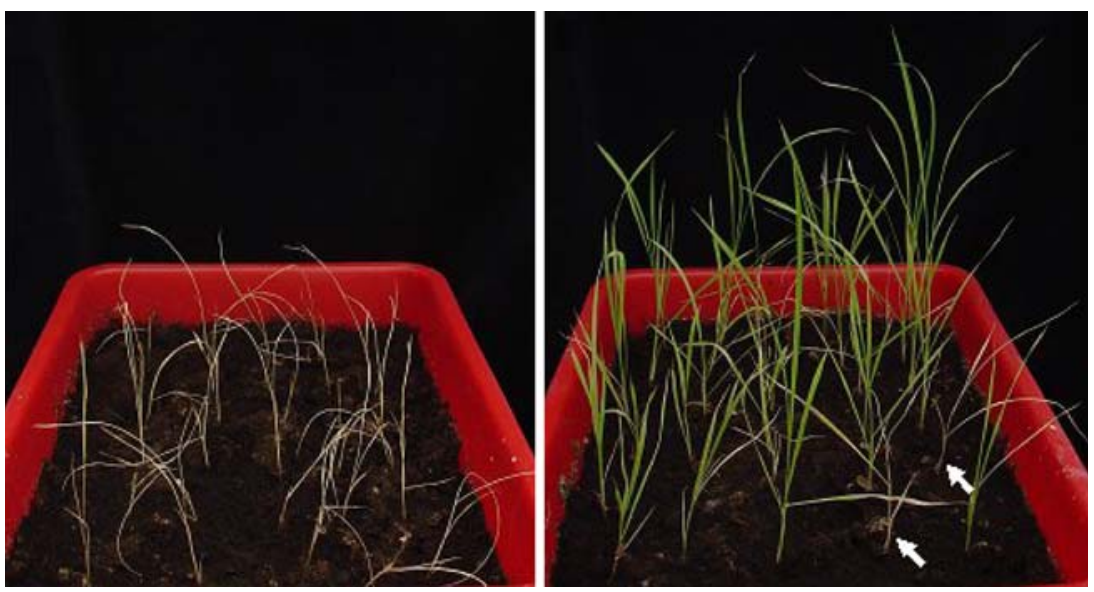

Fig. 6 Glyphosatetolerance analysis of the self-pollinated progeny of $K C E H$ transposed (left) and untransposed line (right). The arrows indicate the null $\mathrm{KCEH}$ progeny, which are not resistant to glyphosate rice. We used glyphosate as the selection agent for rice transformation and found that the proliferation of the transgenic rice calli and subsequent shoot regeneration was as efficient as with hygromycin (Fig. 3). Another indication of a useful selectable marker gene is the copy number of the T-DNA integration sites. In transgenic rice, about $37 \%$ of transgenic rice contains one integrated T-DNA copy harboring hpt (Li et al. 2003), the most frequently used selectable marker gene in rice. Our results with glyphosate used as the selectable agent showed up to $43 \%$ of transgenic rice harboring a single transgene copy (Fig. 4).
Furthermore, transgenic T1 progeny can be screened conveniently by applying commercial Roundup ${ }^{\circledR}$ (Fig. 6). All of these results suggested that epsps could be a suitable selectable marker.

With a transposon system to terminate the marker gene, the GOI-containing transposon could be used to create more independent transgenic lines from one successful transformant. We previously studied the behavior of another PR-1a::TPase-based inducible transposon in rice and found the transposons to be very active in SA-induced rice calli (Charng et al. 2007). We applied the same method to induce T1 rice 
Table 1 Genomic sequences flanking $K E C H$ insertions in transgenic rice plants. The T-DNA integration site of each line is indicated after its designation

\begin{tabular}{llllll}
\hline Line & Chromosome & BACs/PACs & Insertion position (bp) & GenBank accession no. & Identities \\
\hline K-08 (21) & (T-DNA) 10 & OSJNBb0060I05 & 56901 & AC092697 & $86 / 86(100 \%)$ \\
& $($ KCEH) 10 & OSJNBa0040D23 & 32948 & AC074196 & $102 / 102(100 \%)$ \\
K-18 (12) & $($ T-DNA) 3 & OSJNBa0039F10 & 52992 & AC137931 & $127 / 128(99 \%)$ \\
& $($ KCEH) 3 & OSJNBa0045E22 & 156408 & AC137072 & $217 / 217(100 \%)$ \\
& $($ KCEH) 8 & OSJNBb0092C08 & 74311 & AP005391 & $251 / 251(100 \%)$ \\
K-26 & (T-DNA) 4 & OSJNBb0022F23 & 130273 & AL606447 & $227 / 227(100 \%)$ \\
& nd & nd & nd & nd & AC109594 \\
K-34 & (T-DNA) 11 & OSJNBa007P22 & 82765 & AC109594 & $256 / 256(100 \%)$ \\
& $($ KCEH) 11 & OSJNBa0007P22 & 143401 & AC105770 & $198 / 198(100 \%)$ \\
K-40 & (T-DNA) 5 & OJ1362_D02 & 120545 & AC117265 & $308 / 316(97 \%)$ \\
& $($ KCEH) 5 & OJ1281_H05 & 30721 & & $290 / 290(100 \%)$ \\
& & & &
\end{tabular}

nd=no detectable product obtained after TAIL PCR amplification

calli containing $\mathrm{KCEH}$ and found similar results for $16 \%$ somatic and $4.6 \%$ germinal transposition efficiency. Some transposon-containing transgenic lines have high transposition efficiency (Charng et al. 2007) and could be used as starters to create a sufficient number of marker-off transgenic lines. Although we have also observed that the $A c$-based inducible transposons show a preference for linked transposition (Table 1 and Charng, unpublished results), the $\mathrm{KCEH}$ system was designed to truncate the marker gene, with no need for out-crossing.

One concern with the system is that tightly linked transposition can cause the marker gene to remain active. This possibility could be rare in plants. A linked transposition consists of four patterns: up- or down-stream integration plus forward or reverse of the marker's reading frame. Even if a tightly linked, downstream, forward transposition occurs, it can be ruled out by analysis of the flanking DNA sequences or glyphosate-tolerance activity. Indeed, Fig. 6 showed the loss of glyphosate-tolerance function in the progeny of a transposed transgenic rice line harboring the $\mathrm{KCEH}$ system.

Another application of the system of transposonmediated break in the exons of a transgene is to terminate the expression of the transposase gene of $A c$ itself. Since the native $A c$ transposase gene contains four introns, we located one end of $A c$ in the intron of the transposase gene itself to yield an inducible self-stabilizing transposon, which has been useful for creating stable knockout mutants in rice (Charng et al., unpublished results). Therefore, the $A c$ $3^{\prime}$ end can be located in one intron of the native transposase gene, which would be replaced with the TPase, as shown in Fig. 1a. The induced transposition can truncate both epsps and TPase to terminate the marker gene and prevent further transposition. Alternatively, to accommodate a new marker gene into the KCEH system, one could implant another system to eliminate the complicated cloning procedure. Indeed, a Gateway-based system reported by Magnani et al. (2006) for multi-site cloning in one recombination event could imply more opportunities for application of the KCEH system.

Acknowledgments We thank Dr. Y. Kishima (Hokkaido University, Japan) for providing the plasmid. This project was supported by the National Science Council (grant no. NSC932317-B-002-007) of Taiwan.

\section{References}

Bradshaw LD, Padgette SR, Kimball SL, Wells BH (1997) Perspectives on glyphosate resistance. Weed Technol 11:189-198

Charng Y-C, Pfitzner AJP, Pfitzner UM, Charng-Chang K-F, Chen C-M, Tu J, Kuo T-T (2000) Construction of an inducible transposon, INAc, to develop a gene tagging system in higher plants. Mol Breed 6:353-367

Charng Y-C, Wu G, Hsieh C-S, Chuan H-N, Huang J-Y, Yeh L-C, Shieh Y-H, Tu J (2007) The inducible transposon system for rice functional genomics. Bot Stud 48:1-11 
Clemente TE, LaVallee BJ, Howe AR, Conner-Ward D, Rozman RJ, Hunter PE, Broyles DL, Kasten DS, Hinchee MA (2000) Progeny analysis of glyphosate selected transgenic soybeans derived from agrobacterium-mediated transformation. Crop Sci 40:797-803

Ebinuma H, Sugita K, Matsunaga E, Yamakado M (1997) Selection of marker-free transgenic plants using the isopentenyl transferase gene. Proc Natl Acad Sci USA 94:2117-2121

Ebinuma H, Sugita K, Matsunaga E, Endo S, Yamada K, Komamine A (2001) Systems for the removal of a selection marker and their combination with a positive marker. Plant Cell Rep 20:383-392

Endo S, Sugita K, Sakai M, Tanaka H, Ebinuma H (2002) Single-step transformation for generating marker-free transgenic rice using the ipt-type MAT vector system. Plant J 30:115-122

Gruener R, Pfitzner UM (1994) The upstream region of the gene for the pathogenesis-related protein 1a from tobacco responds to environmental as well as to developmental signals in transgenic plants. Eur J Biochem 220:247-255

Hajdukiewicz PTJ, Gilbertson L, Staub JM (2001) Multiple pathways for Cre/lox-mediated recombination in plastids. Plant J 27:161-170

Hare PD, Chua N-H (2002) Excision of selectable marker genes from transgenic plants. Nat Biotechnol 20(6):575580

Haring MA, Rommens CMT, Nijkamp HJJ, Hille J (1991) The use of transgenic plants to understand transposition mechanisms and to develop transposon tagging strategies. Plant Mol Biol 16:449-461

Hohn B, Levy AA, Puchta H (2001) Elimination of selection markers from transgenic plants. Curr Opin Biotechnol 12:139-143

Howe AR, Gasser CS, Brown SM, Padgette SR, Hart J, Parker GB, Fromm ME, Armstrong CL (2002) Glyphosate as a selective agent for the production of fertile transgenic maize (Zea mays L.) plants. Mol Breed 10:153-164

Hu T, Metz S, Chay C, Zhou HP, Biest N, Chen G, Cheng M, Feng X, Radionenko M, Lu F, Fry J (2003) Agrobacterium-mediated large-scale transformation of wheat (Triticum aestivum L.) using glyphosate selection. Plant Cell Rep 21:1010-1019
Li F, Dey M, He C, Sangwan V, Wu X, Wu R (2003) Rapid PCR-based determination of transgene copy number in rice. Plant Mol Biol Rep 21:73-80

Liu YG, Mitsukawa N, Oosumi T, Whittier RF (1995) Efficient isolation and mapping of Arabidopsis thaliana T-DNA insert junctions by thermal asymmetric interlaced PCR. Plant J 8:457-463

Magnani E, Bartling L, Hake S (2006) From Gateway to MultiSite Gateway in one recombination event. BMC Mol Biol 7:46. doi:10.1186/1471-2199-7-46

Miki B, McHugh S (2004) Selectable marker genes in transgenic plants: applications, alternatives and biosafety. J Biotechnol 107:193-232

Ow DW (2007) GM maize from site-specific recombination technology, what next? Curr Opin Biotechnol 18:115-120

Sambrook J, Russel DW (2001) Molecular cloning: a laboratory manual, 3rd edn. Cold Spring Harbor, New York

Schönbrunn E, Eschenburg S, Shuttleworth WA, Schloss JV, Amrhein N, Evans JNS, Kabsch W (2001) Interaction of the herbicide glyphosate with its target enzyme 5-enolpyruvylshikimate 3-phosphate synthase in atomic detail. Proc Natl Acad Sci USA 98:1376-1380

Sha Y, Li S, Pei Z, Luo L, Tian Y, He C (2004) Generation and flanking sequence analysis of a rice T-DNA tagged population. Theor Appl Genet 108:306-314

Toki S (1997) Rapid and efficient Agrobacterium-mediated transformation in rice. Plant Mol Biol Rep 15:16-21

van Loon LC, van Kammen A (1970) Polyacrylamide disc electrophoresis of the soluble leaf proteins from Nicotiana tabacum var. "Samsun" and "Samsun NN". II. Changes in protein constitution after infection with tobacco mosaic virus. Virology 40:199-211

White RF (1979) Acetylsalicylic acid (aspirin) induces resistance to tobacco mosaic virus in tobacco. Virology 99:410-412

Zhou H, Arrowsmith JW, Fromm ME, Hironaka CM, Taylor ML, Rodriguez D, Pajeau ME, Brown SM, Santino CG, Fry JE (1995) Glyphosate-tolerant CP4 and GOX genes as a selectable marker in wheat transformation. Plant Cell Rep 15:159-163

Zuo JR, Niu Q-W, Møller SG, Chua N-H (2001) Chemicalregulated, site-specific DNA excision in transgenic plants. Nat Biotechnol 19:157-161 